DOI: 10.12731/2227-930X-2019-1-51-58

УДК 004.94

\title{
АЛГОРИТМИЗАЦИЯ РЕШЕНИЙ, БАЗИРУЮЩИХСЯ НА ПОВЫШЕНИИ РЕСУРСОЭФФЕКТИВНОСТИ ОРГАНИЗАЦИЙ, СВЯЗАННЫХ С ПЕРЕВОЗКАМИ
}

\section{Львович Я.Е., Преображенский А.П., Чопоров О.Н.}

В данной статье рассматриваются вопросы, связанные с исследованием вопросов повышения ресурсоэффективности перевозок в организачиях. Предложена оптимизационная модель. Приведен пример расчетов.

Ключевые слова: система перевозок; ресурсоэффективность; оптимизачия; моделирование; организачия.

\section{ALGORITHMIC SOLUTIONS BASED ON THE RESOURCE-EFFICIENCY OF THE ORGANIZATIONS CONNECTED WITH TRANSPORT}

\section{Lvovich Ya. E., Preobrazhenskiy A.P., Choporov O.N.}

In this paper the questions connected with research of questions of increase of resource efficiency of transportations in the organizations are considered. An optimization model is proposed. An example of calculations is given.

Ключевые слова: transportation system; resource efficiency; optimization; modeling; organization.

Введение. При управлении процессами перевозок в организациях необходимо стремиться к повышению параметров ресурсоэффективности. Для того, чтобы выработать вариант возможного планирования, требуется, чтобы была задана функция, показыва- 
ющая как меняется ресурсоэффективность в течение периода планирования $[1,2]$. То, как такая функция задается определяет вид структуры оптимизационных моделей и алгоритмических процедур решения задачи оптимизации. Когда мы ищем многошаговое решение по всему периоду планирования, то приходится сталкиваться с неоднозначностью того, как исполняются экстремальные и граничные требования. При этом получается большое число вариантов управленческих решений $[3,4]$.

Вследствие того, что есть не один вариант, необходимо привлекать экспертные оценки с тем, чтобы было принято окончательное решение. За счет оценок групп экспертов есть возможности для ранжирования вариантов относительно значимости. При этом не только растет ресурсоэффективность, но учитывается опыт и мнения экспертов относительно улучшения работы организации [5], достижения более высоких уровней качества перевозок. Также существуют возможности для анализа вариантов, чтобы организация в соответствующих рейтингах смогла занимать более высокие позиции. Тогда необходимо, чтобы позиция выбиралась на основе оптимизационно-экспертного подхода [6], и она достигается при соответствующих условиях роста ресурсоэффективности перевозок.

Чтобы подсистема интеллектуальной поддержки принятия решений эффективным образом функционировала, необходимо, чтобы была оптимизация по распределению дополнительных затрат, связанных с достижением условий роста ресурсоэффективности организации и получение окончательного экспертного оценивания. Тогда проведение верификации по оптимальному характеру управленческих решений ведется за счет того, что сравнивается рейтинг организации и рейтинговые оценки работы соответствующих организаций.

Описание оптимизационной модели. Пусть функция изменения ресурсоэффективности перевозок задается двумя способами на периоде планирования $\mathrm{T}+\tau$, здесь $\tau=\overline{1, \Delta}$ показывают номера временных интервалов: 
- как зависимость роста ресурсоэффективности перевозок от роста затрат в течение временных интервалов $\tau=\overline{1, \Delta}$;

- за счет того, что последовательным образом задаются значения ресурсоэффективности перевозок, соответствующие временным интервалам.

Предполагаем, что на базе модели

$$
H=f\left(x_{1}, x_{2}, t\right)
$$

можно сделать прогнозирование по ресурсоэффективности перевозок на периоде планирования $T+\tau, \tau=\overline{1, \Delta}$. В (1) $x_{1}-$ показатели, которые влияют на изменения расходов в организации, $x_{2}-$ объемы затрат, обеспечивающие рост доходов и рост ресурсоэффективности.

Они характеризуются значениями внутри интервал $0 \leq x_{2} \leq X$, $\tau=\overline{1, \Delta}$. В этом случае для каждого значения $\tau$ и $x_{2 \tau}$ из соответствующих интервалов на базе (1) идет вычисление значений функции

$$
f\left(x_{2}, t\right), \forall \tau=\overline{1, \Delta} \text {. }
$$

Функции (2) по периодам планирования показывают, как меняется ресурсоэффективность перевозок по интервалам $\tau=\overline{1, \Delta}$. Можно, анализируя особенности изменения ресурсоэффективности, на основе экспертного подхода использовать функции, демонстрирующие ускоренное изменение ресурсоэффективности. При помощи таких функций оптимизируются объемы, ведущие к росту доходов, так, чтобы не был превышен определенные объемы расходов $X$

$$
\sum_{\tau}^{\Delta} x_{2 \tau} \leq X
$$

Пусть функции, связанные с ускоренным изменением обозначаются $f 2\left(x_{2 \tau}\right)$. Они показывают значения функции (2), которые задаются для изменений значений $x_{2 \tau}$ по диапазону $0 \leq x_{2 \tau} \leq X$

Можно задать относительные значения переменных $x 2_{2 \tau}$ и функции (3):

$$
f 2(\tau)=\frac{f(\tau)-f(\Delta)}{f(\Delta)} \cdot 100 \%,
$$




$$
x_{2 \tau}=\frac{X-x_{2 \tau}}{x_{2 \tau}} \cdot 100 \%,
$$

где $f(\Delta)$ - показывает ресурсоэффективность по перевозкам, которую достигает организация, когда завершается $\Delta$-й временной интервал.

Переменные (4) будут целочисленными, когда ищется оптимальный объем затрат, внутри интервала

$$
0 \leq x_{2 \tau} \leq X, \tau=\overline{1, \Delta}
$$

Осуществляется прогноз того, как изменяется функция (5) на базе принятия решения по интервалу

как $S$-образная функция [7].

$$
0 \leq f 2(\tau) \leq F
$$

Эксперт осуществляет прогнозирование того, что для начала интервала (6) функция (5) изменяется так, что это соотносится с прогностической моделью (1). Потом идет задание ускоренного изменения. К концу интервала (6) рост доходов будет частичным образом компенсирован за счет уровня затрат, скорость роста $2(\tau)$ будет снижена.

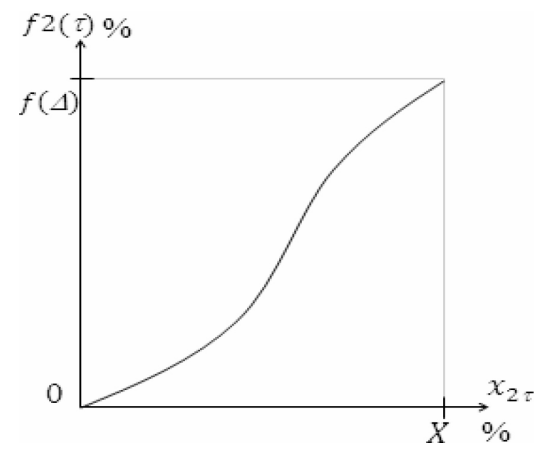

Рис. 1. Иллюстрация $S$-образной функции, демонстрирующей, как в \% меняется ресурсоэффективность при росте объемов затрат, ведущих к росту доходов

Тогда на основе задания (6), (7) и связи $[8,9]$ ресурсоэффективности по перевозкам и роста увеличения затрат можно сделать оценку оптимальных объемов затрат, ведущих к росту доходов. Тогда максимизируется общий рост эффективности по всем временным интервалам: 


$$
\sum_{\tau}^{\Delta} f\left(x_{2 \tau}\right) \rightarrow \max _{x_{2 \tau}, \tau=1,4}
$$

Учитывается также ограничение (2) и неотрицательность $x 2_{2 \tau}$. Получается задача сепарабельного программирования.

\section{Результаты расчетов}

Предположим, что рассматривается 3 временных интервала. При решении задачи оптимизации определяются 3 значения $x 2_{2 \tau}$, когда задаются $X=5,8 \%, f(\Delta)=6,8 \%$, получаются $S$-образные функции. Тогда строится оптимизационная модель:

$$
\begin{gathered}
f\left(x 2_{21}\right)+f\left(x 2_{22}\right)+f\left(x 2_{23}\right) \underset{x 2_{21}, x 2_{22}, x 2_{23},}{\max }, \\
x 2_{21}, x 2_{22}, x 2_{23}=6, \\
x 2_{21}, x 2_{22}, x 2_{23}=0,1,2,3,4,5,6 .
\end{gathered}
$$

Для каждого $i$-го шага есть свои состояния $d_{i}$ анализируемой системы, которые от одного временного интервала к другому меняются.

Ниже в таблице показано, как приходим к оптимальному решению.

\begin{tabular}{|c|c|c|c|c|c|c|c|c|}
\hline \multirow{2}{*}{1 интервал } & $d_{i}$ & 0 & 1 & 2 & 3 & 4 & 5 & 6 \\
\cline { 2 - 9 } & $x 2_{2 i}\left(d_{i}\right)$ & 0 & 1 & 2 & 3 & 4 & 5 & 6 \\
\hline \multirow{2}{*}{2 интервал } & $d_{i}$ & 0 & 1 & 2 & 3 & 4 & 5 & 6 \\
\cline { 2 - 9 } & $x 2_{2 i}\left(d_{i}\right)$ & 0 & 1 & 2 & 3 & 4 & 5 & 6 \\
\hline \multirow{2}{*}{3 интервал } & $d_{i}$ & 0 & 1 & 2 & 3 & 4 & 5 & 6 \\
\cline { 2 - 9 } & $x 2_{2 i}\left(d_{i}\right)$ & 0 & $0 ; 1$ & $0 ; 1$ & 0 & 0 & $0 ; 1$ & $0 ; 1$ \\
\hline
\end{tabular}

Мы выбираем, как оптимальное, первое значение, $x 2{ }_{26}$ при этом состояние системы $d_{3}=6$.

С тем, чтобы окончательным образом сделать выбор варианта по множеству возможных решений необходимо, чтобы была привлечена группа экспертов. Каждым из экспертов устанавливается своя последовательность вариантов, идущих от худших к лучшим.

\section{Выводы}

Когда повышается ресурсоэффективность перевозок организации, то целесообразно применять экспертно-оптимизационные 
способы в комбинации с планированием по заданным временным интервалам. На основе оптимизационной модели есть возможность для определения совокупности вариантов того, как распределяются затраты по планируемым временным интервалам, что определяется тем, как меняется функция ресурсоэффективности перевозок.

\section{Сиисок литературы}

1. Калиберда Е.А., Христосова Н.Г. Автоматизация деятельности отдела доставки заказов интернет-магазина // International Journal of Advanced Studies. 2018. T. 8. № 1-2. C. 65-72.

2. Тюльпинова Н.В. Программный модуль для имитационного моделирования предприятий розничной торговли // International Journal of Advanced Studies. 2018. T. 8. № 1-2. C. 187-192.

3. Юрочкин А.Г., Коростелева Н.А. Анализ особенностей управления и менеджмента в современных компаниях // Вестник Воронежского института высоких технологий. 2018. № 3(26). С. 78-80.

4. Юрочкин А.Г., Коростелева Н.А. Вопросы обеспечения стратегического управления организации // Вестник Воронежского института высоких технологий. 2018. № 3(26). С. 135-138.

5. Гоян В.И., Никонова Е.3. Реинжиниринг и его место в жизненном цикле информационной системы // International Journal of Advanced Studies. 2019. Т. 9. № 1-2. С. 45-51.

6. Львович И.Я. Львович Я.Е., Фролов В.Н. Информационные технологии моделирования и оптимизации: краткая теория и приложения. Воронеж: ИПЦ «Научная книга», 2016. 444 с.

7. Батищев Д.И., Львович Я.Е., Фролов В.Н. Оптимизация в САПР. М.: Высш.шк., 1977. 416 с.

8. Зазулин А.В., Преображенский Ю.П. Особенности построения семантических моделей предметной области // Вестник Воронежского института высоких технологий. 2008. № 3. С. 026-028.

9. Зяблов Е.Л., Преображенский Ю.П. Построение объектно-семантической модели системы управления // Вестник Воронежского института высоких технологий. 2008. № 3. С. 029-030. 


\section{References}

1. Kaliberda E.A., Hristosova N.G. International Journal of Advanced Studies. 2018. V. 8. № 1-2, pp. 65-72.

2. Tyul'pinova N.V. International Journal of Advanced Studies. 2018. V. 8. № 1-2, pp. 187-192.

3. Yurochkin A.G., Korosteleva N.A. Vestnik Voronezhskogo instituta vysokih tekhnologij. 2018. № 3(26), pp. 78-80.

4. Yurochkin A.G., Korosteleva N.A. Vestnik Voronezhskogo instituta vysokih tekhnologij. 2018. № 3(26), pp. 135-138.

5. Goyan V.I., Nikonova E.Z. International Journal of Advanced Studies. 2019. V. 9. № 1-2, pp. 45-51.

6. L'vovich I.Ya. L'vovich Ya.E., Frolov V.N. Informacionnye tekhnologii modelirovaniya i optimizacii: kratkaya teoriya i prilozheni$y a$ [Information technology modeling and optimization: a brief theory and applications]. Voronezh: IPC "Nauchnaya kniga", 2016. $444 \mathrm{p}$.

7. Batishchev D.I., L'vovich Ya.E., Frolov V.N. Optimizaciya v SAPR [Optimization in CAD]. M.: Vyssh.shk., 1977. 416 p.

8. Zazulin A.V., Preobrazhenskij Yu.P. Vestnik Voronezhskogo instituta vysokih tekhnologij. 2008. № 3, pp. 026-028.

9. Zyablov E.L., Preobrazhenskij Yu.P. Vestnik Voronezhskogo instituta vysokih tekhnologij. 2008. № 3, pp. 029-030.

\section{ДАННЫЕ ОБ АВТОРАХ}

Львович Яков Евсеевич, доктор технических наук, профессор Федеральное государственное образовательное учреждение высшего образования «Воронежский государственный технический университет»

ул. 20 лет Октября, 84, г. Воронеж, 394006, Российская Федерачия

Komkovvivt@yandex.ru

Преображенский Андрей Петрович, профессор, доктор технических наук, доцент 
Автономная некоммерческая образовательная организачия высшего образования "Воронежский институт высоких технологий»

ул. Ленина, 73а, Воронеж, 394043, Российская Федераџия Komkovvivt@yandex.ru

Чопоров Олег Николаевич, профессор, доктор технических наук, профессор Федеральное государственное образовательное учреждение высшего образования «Воронежский государственный технический университет» ул. 20 лет Октября, 84, г. Воронеж, 394006, Российская Федерачия

Komkovvivt@yandex.ru

\section{DATA ABOUT THE AUTHORS}

Lvovich Yakov Yevseevich, Doctor of Technical Sciences, Professor Voronezh State Technical University 84, 20 let Oktyabrya Str., Voronezh, 394006, Russian Federation Komkovvivt@yandex.ru

ORCID: 0000-0002-7051-3763

Preobrazhenskiy Andrey Petrovich, Doctor of Technical Sciences, Professor, Associate Professor Voronezh Institute of High Technologies 73a, Lenin Str., Voronezh, 394043, Russian Federation Komkovvivt@yandex.ru ORCID: 0000-0002-6911-8053

Choporov Oleg Nikolaevich, Doctor of Technical Sciences, Professor Voronezh State Technical University 84, 20 let Oktyabrya Str., Voronezh, 394006, Russian Federation Komkovvivt@yandex.ru ORCID: 0000-0002-3176-499X 